\title{
EFEITO DO BARBEXACLONE NAS CRISES CONVULSIVAS DE DIFICIL CON'ROLE
}

\author{
JOSE MAURO BRAZ DE LIMA* \\ CLOVIS OLIVEIRA * \\ LUIZ ANTONIO ALVES DURO *** \\ NATALIA DE MESQUITA POIARES ***
}

Por vezes defrontamo-nos com pacientes que apresentam crises convulsivas de difícil controle terapêutico, constituindo-se em um problema importante na clínica neurológica. A gravidade desta situação é vista sob vários aspectos: integração social e familiar do paciente, repercussões psicológicas para o próprio indivíduo e, o mais importante, lesões cerebrais decorrentes das crises repetidas que ueixam sequelas e agravam, sobremaneira, o estado mórbido do paciente. so por esses aspectos torna-se imperiosa a busca de medicamentos ou processos terapêutıcos que visem a mınimızar ou anular este sério problema que são as "crises de difícil controle". Estas são ao nosso ver definidas como crises epilépticas, geralmente tipo grande-mal, de etiologias várias, que são rebeldes à terapêutica convencional, isto é, medicamentos antiepilépticos já consagrados pelo uso. Atastamos inicialmente problemas de dosagens inadequadas, falha nas tomadas pelo próprio paciente e outros fatores externos ou internos que possam interferir na gênese das crises (doenças sistêmicas, por exemplo).

O objetivo deste trabalho é demonstrar a eficácia do barbexaclone isolado ou associado, nestes casos, principalmente com a acetazolamida. A vantagem do primeiro é a associação do fenobarbital a um estimulante do sistema nervoso central (ciclo-hexil-metil-aminopropano-CHP), o que permite a utilização de doses úteis do fenobarbital sem o inconveniente efeito colateral da sonolência, evitando-se ainda a depressão da vigilia, prejudicial nas atividades que o paciente deve exercer em sua vida diária $1,2,3,4,5,7$.

\section{MATERIAL E METODOS}

Selecionamos 11 pacientes que vinham fazendo tratamento com diversos medicamentos sem bons resultados. Este ensaio visa apenas ao controle de. crises convulsivas tipo grande-mal, presentes nos 11 casos, com alguns apresentando outros tipos associados. Nesses casos, em que havia crises tipo grande-mal e tónicas associadas, somávamos - número dírío das mesmas, para fins estatísticos. As crises de pequeno-mal foram

Trabalho realizado no Instituto de Neurologia Deolindo Couto (Servico do Prof. Bernardo Couto): * Prof. Auxiliar de Ensino de Neurologia da Faculdade de Medicina da Universidade Federal do Rio de Janeiro (FM-UFRJ); ** Prof. Adjunto de Neurologia da FM-UFTJ; *** Mestrando em Neurologit do INDC-UFRJ; **** Médica residente do INDC-UFRJ. 
desprezadas. Em 8 casos foi necessária a internaçăo devido à gravidade do quadro. Após um período variável de dias ou semanas, quando já tínhamos algum controle das crises, estes pacientes passavam para tratamento ambulatorial.

A idade de início das crises variou de 3 meses a 42 anos de idade $e$ início do tratamento variou de 5 a 46 anos (Tabela 1). Quanto ao sexo, 4 eram do sexo feminino e 7 do masculino. O tempo de tratamento especifico variou de 3 a 39 meses. No caso 4 observamos a medicação por apenas 3 meses pois que não foi boa a sua resposta. A dose utilizada nas 24 horas do barbexaclone variou de 75 a $400 \mathrm{mg}$, com dose média de $279 \mathrm{mg}$ (Tabela 1).

\begin{tabular}{|c|c|c|c|c|c|c|c|}
\hline Caso & Idade & Sexo & $\mathbf{T P}$ & IN & Medicação & Assoc. & T.T. \\
\hline 1 & $8 \mathbf{a}$ & $\mathbf{M}$ & $\mathbf{G M}+\mathbf{T}$ & $3 m$ & $200 \mathrm{mg}$ & Acetazol. & $13 \mathrm{~m}$ \\
\hline 2 & $14 u$ & $\mathbf{F}$ & $\mathbf{G M}+\mathbf{P M}$ & $5 a$ & $300 \mathrm{mg}$ & Acetazol. & $13 \mathrm{~m}$ \\
\hline 3 & $12 u$ & $\mathbf{F}$ & $\mathbf{G M}$ & $9 \mathrm{a}$ & $400 \mathrm{mg}$ & Fenobarb. & $39 \mathrm{~m}$ \\
\hline 4 & $18 \mathrm{a}$ & $\mathbf{F}$ & GM & $15 a$ & $200 \mathrm{mg}$ & Fenobarb. & $3 \mathrm{~m}$ \\
\hline 5 & $10 \mathbf{a}$ & $\mathbf{M}$ & $\mathbf{G M}+\mathbf{P M}+\mathbf{T}$ & $2 a$ & $250 \mathrm{mg}$ & Acetazol. & $9 \mathrm{~m}$ \\
\hline 6 & $24 a$ & $\mathbf{M}$ & GM & $22 \mathrm{a}$ & $300 \mathrm{mg}$ & Acetazol. & $12 \mathrm{~m}$ \\
\hline 7 & $46 a$ & $\mathbf{M}$ & GM & $42 a$ & $300 \mathrm{mg}$ & Acetazol. & $11 \mathrm{~m}$ \\
\hline 8 & $21 a$ & $\mathbf{M}$ & GM & $15 a$ & $400 \mathrm{mg}$ & Acetazol. & $13 \mathrm{~m}$ \\
\hline 9 & $11 \mathrm{a}$ & $\mathbf{F}$ & $\mathbf{G M}+\mathbf{T}$ & $7 a$ & $300 \mathrm{mg}$ & & $7 \mathrm{~m}$ \\
\hline 10 & $5 \mathbf{a}$ & $\mathbf{M}$ & $\mathbf{G M}+\mathbf{T}$ & $5 \mathbf{a}$ & $350 \mathrm{mg}$ & - & $7 \mathrm{~m}$ \\
\hline 11 & $9 m$ & $\mathbf{M}$ & GM & $3 \mathrm{~m}$ & $75 \mathrm{mg}$ & Nitrazep. & $6 \mathrm{~m}$ \\
\hline
\end{tabular}

Tabela 1 - Material de estudo: Idade = idade do paciente wo iniciar a medicaså; IN $=$ idade de inicio da doenga; A8soc. $=$ medicamento associado; $T . T .=$ tempo de tratamento; $a=$ anos; $m=$ meses; $H^{\top}=$ feminino; $M=$ masculino; $T P=$ tipo de crise; $G M=$ grande mal; $T=$ crises tonicas; $P M=$ crises de pequeno mal; Acetazol. = acetazolamida; Frenobarb. = fenobarbital.

Os pacientes foram submetidos a exame de rotina constando de hemograma completo, sorologia para lues (VDRL), glicemia, azotemia, exame sumário de urina e parasitológico de fezes. EEG e exames radiológicos de crânio nas incidências comuns foram feitos em todos os pacientes.

Quanto ao esquema posológico, 2 pacientes fizeram uso exclusivo de barbexaclone * (casos 9 e 10); em 6 pacientes associados a acetazolamida foi usada complementarmente (casos 1, 2, 5, 6, 7, 8); em 2 outros casos acrescentamos fenobarbital na dose de 50 a $100 \mathrm{mg}$, à noite (Tabela 1). Em nenhum destes casos observamos efeitos colaterais de grande importancia que obrigassem a suspensão do tratamento. Leve agitaçăo ou insónia presentes em alguns casos desapareceram com a continuidade do tratamento ou modificação no horário das tomadas. Em relaçăo a fatores etiopatogênicos, observamos 4 casos de traumatismo craniano, 4 de sindrome de Lennox-Gastaut, 3 casos com história de traumatismo de parto (asfixia neonatal). Em 3 pacientes não conseguimos determinar a possivel causa etiologica.

* Barbexaclone (Maliasin) fornecido por Knoll S/A Produtos Químicos e Farmacêuticos. 


\title{
RESULTADOS
}

\begin{abstract}
As causas etiológicas apontadas na Tabela 1 são consideradas como prováveis, pois que o quadro mórbido iniciou-se após as mesmas, exceto os casos de asfixia. $O$ caso 6 foi devido a queda de trem tendo o paciente sofrido traumatismo cranioencefálico com afundamento frontal que motivou a lobectomia frontal bilateral. Observamos aqui que os traumatismos cranianos são importantes na genese de casos de epilepsias sintomáticas (36\%). Utilizando como parametro clínico o "escore" dado pela frequencia e intensidade das crises, notamos que ocorreram casos de melhoras espetaculares (casos 5, 9 e 10). Assim sendo, consideramos como resultado muito bom (MB) ou excelente os obtidos em 7 casos (64\%), bom (B) em dois casos (18\%) e nulo (N) em dois casos (18\%). Podemos considerar como resultados bastante eficazes (MB e B) $82 \%$ dos casos (Tabela 3).
\end{abstract}

Efeitos colaterais

\begin{tabular}{lll}
\hline \hline Agitaçå & 3 & casos \\
Anorexia & 1 & caso \\
Agressividade & 1 & caso \\
Insônia & 1 & caso \\
\hline
\end{tabular}

Tabela 2 - Efeitos colaterais apresentados por 6 pacientes.

\begin{tabular}{lrr}
\hline Resultados & $\begin{array}{c}\text { Número de } \\
\text { pacientes }\end{array}$ \\
\hline Muito bom & 7 & $64 \%$ \\
Bom & 2 & $18 \%$ \\
Regular & 0 & $0 \%$ \\
Nulo & 2 & $18 \%$ \\
Piora & 0 & $0 \%$ \\
\hline
\end{tabular}

Tabela s- Resultodas obtidos nos 11 pacientes.

\section{COMENTARIOS}

E interessante notar que apenas 2 pacientes que fizeram uso de barbexaclone estavam no grupo $\mathrm{N}$. E nossa opinião que a associação do barbexaclone $\mathrm{e}$ acetazolamida é realmente eficaz em casos de difícil controle, e deve, portanto, ser experimentada. Um item especial deve ser reservado para a forma de introduzir o medicamento. Como referido em outro trabalho ${ }^{6}$, o binômio, horário da crise-horário da medicação deve ser observado. Com isso, alcançamos bons resultados, com doses relativamente baixas e efeitos colaterais insignificantes. Baseados unicamente em observações clínicas, acreditamos que o barbexaclone atinge o efeito terapêutico máximo, 2 horas após ingerido. Permanece com essa ação durante 4 a 6 horas, decaindo a seguir. Assim sendo, devemos fazer um mapa referente ao horário das crises dos pacientes. Obtemos assim o horário em que as crises são mais frequentes; a seguir, introduzimos a medicação 2 horas antes desse horário. Assim sendo, o barbexaclone inicia seu efeito terapêutico máximo em cima do horário em que as crises são mais frequentes. A acetazolamida deve ser tomada 2 horas após a tomada do barbexaclone.

Outra importante observação que devemos fazer, conforme já referido, é quanto aos efeitos colaterais de pouca importância (Tabela 2). Como costuma acontecer nos casos em que a dose de fenobarbital é alta para controlar crises convulsivas rebeldes, a sonolência e depressão sensorial ocorreu com relativa frequência. Ora, se por um lado controlam-se as crises, por outro, restringe-se 
o) paciente em suas atividades diárias, () que é altamente danoso. Com 0 barbexaclone isolado, introduzido conforme referido ou associado, principalmente com a acetazolamida, estes dois efeitos colaterais acıma estão, ou ausentes ou bastante diminuidos, podendo-se, portanto, utilizar a dose necessária a fim de controlar as crises ditas de difícil controle. Acreditamos por fim que é válida a indicação do barbexaclone, puro ou associado, nestes casos, preferentemente com a acetazolamida, como droga preferencial nos casos de crises convulsivas de dificil controle nos quais já se fizeram tentativas terapêtica com pouco sucesso.

\section{RESUMU}

Fioi feito ensaio terapêutico visando ao controle de crises rebeldes com o barbexaclone, sendo obtidos resultados bastante positivos. Os autores chamam a atenção para a associação do barbexaclone com a acetazolamida em alguns casos, com bons resultados. Ressaltam a vantagem do medicamento quanto aos efeitos colaterais, observando que com o barbexaclone estes estão diminuídos ou mesmo abolidos, mantendo-se o paciente desperto e participante.

\section{SUMMARY}

Therapeutic effect of barbexaclone in the treatment of refractory epilepsy.

A therapeutic trial using barbexaclone in uncontrolled seizures was undertaken showing favourable results. Special reference to the association of barbexaclone with acetazolamide with good results in many cases is made. Collateral effects are minimal in pacients using barbexaclone.

\section{REFERENCIAS}

1. BECKER, B. - Erfahrungen mit dem Antiepileptikum Muliasin. Med. Welt. 9:577, 1968.

2. CANGER, R. \& WAHL, L. - Die Behandlung der Epilepsie mit Maliasin. Nervenarzt $39: 476,1968$.

3. KRUEGER, H. J. \& SCHWARZ, H. - Klinische Mitteilung zur Epilepsie: Therapie mit Maliasin. Med. Welt. 14:690, 1965.

4. OLIVEIRA, C.: FERNANDES, I. \& LIMA, J. M. - Ensaio clinico com barbexaclone nas epilepsias. Arq. Neuro-Psiquiat. (São Paulo) $35: 68,1977$.

5. OLIVEIRA, C.: FERNANDES, I. \& LIMA, J. M. - Reavaliação do barbexaclone em 20 pacientes epilépticos. Arq. Neuro-Psiquiat. (Såo Paulo) 36:135, 1978.

6. OliveirA, C.; FernaNdeS, I.; LIMA, J. M.; DURO, L. A. A. \& SILVA, C. V. - Efeitos do barbexaclone na sindrome de Lennox-Gastaut. Arq. Neuro-Psiquiat. (São Paulo) $36: 340,1978$.

7. PENIN, H. - Wirkung und Indikation eines neuen Antiepileticums. Dtsch. Med. Wschr. $89: 1683,1964$.

Instituto de Neurologia Deolindo Couto - Av. Venceslau Brds 95, Botafogo 28890 Rio de Janeiro, $R J$ - Brasil. 